\title{
Consumo alimentar, estilo e qualidade de vida de professores do Ensino Técnico
}

\author{
Food consumption, style and quality of life of technical Education Teachers \\ Consumo de alimentos, estilo y calidad de vida de los profesores de Educación Técnica
}

Keytiani Secundo Duarte Landim

ORCID: https://orcid.org/0000-0002-6020-1897 Centro Universitário Adventista de São Paulo, Brasil E-mail: keytiani@hotmail.com

Eloise Cristiane da Silva Oliveira

ORCID: https://orcid.org/0000-0001-9165-9991 Centro Universitário Adventista de São Paulo, Brasil E-mail: eloisecristiane@ gmail.com

Luciana Marcolino Cavalcante

ORCID: https://orcid.org/0000-0001-9543-7288 Centro Universitário Adventista de São Paulo, Brasil

E-mail: luciana.mcavalcante@gmail.com

Vitoria Maximo

ORCID: https://orcid.org/0000-0002-3275-2800 Centro Universitário Adventista de São Paulo, Brasil E-mail: vitoriamaximo@outlook.com

Jessica Matos de Souza

ORCID: https://orcid.org/0000-0002-8111-8611 Centro Universitário Adventista de São Paulo, Brasil E-mail: jessicamatos148@yahoo.com.br

Leslie Andrews Portes

ORCID: https://orcid.org/0000-0003-0537-4725 Centro Universitário Adventista de São Paulo, Brasil

E-mail: leslie.portes@unasp.edu.br

Nyvian Alexandre Kutz

ORCID: https://orcid.org/0000-0003-3905-9061 Centro Universitário Adventista de São Paulo, Brasil

E-mail: nyviankutz@hotmail.com

Narcisio Rios Oliveira

ORCID: https://orcid.org/0000-0001-6981-2988 Centro Universitário Adventista de São Paulo, Brasil E-mail: narcisiorios@gmail.com

Rubia Maria da Penha Mantuani Guimarães

ORCID: https://orcid.org/0000-0002-2656-5662 Centro Universitário Adventista de São Paulo, Brasil E-mail: rubiampmg@hotmail.com

Marcia Maria Hernandes Abreu de Oliveira Salgueiro

ORCID: https://orcid.org/0000-0001-6349-7219

Centro Universitário Adventista de São Paulo, Brasil

E-mail: marciasalgueironutricionista@yahoo.com.br

\section{Resumo}

Este trabalho objetivou analisar o perfil de saúde de professores de uma Escola Técnica Estadual da grande São Paulo. Trata-se de estudo transversal descritivo com professores, por meio de questionário contendo as variáveis sociodemográficas, do consumo alimentar e outros hábitos de vida, conhecimento em nutrição, estilo e qualidade de vida. A amostra foi composta por 43 professores e verificou-se o predomínio de classe socioeconômica B e formação em nível de especialização. O estilo e qualidade de vida foram classificados como bons. O consumo alimentar necessita melhorar e o conhecimento em nutrição foi classificado como moderado. Houve correlações positivas e fracas, mas significantes, entre a idade, a classificação socioeconômica e os escores médios no domínio psicológico da qualidade de vida; entre o estilo de vida e a qualidade de vida geral e nos domínios psicológico e social; assim como correlações fracas e negativas entre a classificação socioeconômica e o domínio social da qualidade de vida.

Palavras-chave: Professor; Consumo alimentar; Qualidade de vida; Estilo de vida; Promoção da saúde.

\section{Abstract}

This study aimed to analyze the health profile of teachers at a State Technical School in the greater São Paulo. This is a descriptive cross-sectional study with teachers, using a questionnaire containing the socio-demographic variables, 
food consumption and other lifestyle habits, knowledge of nutrition, style and quality of life. The sample consisted of 43 teachers and there was a predominance of socioeconomic class B and training at a specialization level. The style and quality of life were classified as good. Food consumption needs to improve and knowledge of nutrition was classified as moderate. There were positive and weak correlations, but significant, between age, socioeconomic classification and average scores in the psychological domain of quality of life; between lifestyle and general quality of life and in the psychological and social domains; as well as weak and negative correlations between socioeconomic classification and the social domain of quality of life.

Keywords: Teacher; Food consumption; Quality of life; Lifestyle; Health promotion.

\section{Resumen}

Este trabajo tuvo como objetivo analizar el perfil de salud de los docentes de una Escuela Técnica del Estado en el Gran São Paulo. Se trata de un estudio descriptivo transversal con docentes, mediante un cuestionario que contiene las variables sociodemográficas, consumo de alimentos y otros hábitos de vida, conocimientos de nutrición, estilo y calidad de vida. La muestra estuvo conformada por 43 docentes y hubo predominio de clase socioeconómica B y formación a nivel de especialización. El estilo y la calidad de vida se clasificaron como buenos. El consumo de alimentos necesita mejorar y el conocimiento de nutrición se clasificó como moderado. Hubo correlaciones positivas y débiles, pero significativas, entre edad, clasificación socioeconómica y puntajes promedio en el dominio psicológico de la calidad de vida; entre el estilo de vida y la calidad de vida en general y en los dominios psicológico y social; así como correlaciones débiles y negativas entre la clasificación socioeconómica y el dominio social de la calidad de vida.

Palabras clave: Profesor; Consumo de comida; Calidad de vida; Estilo de vida; Promoción de la salud.

\section{Introdução}

A Carta de Ottawa para Promoção da Saúde (PS) sugere que a saúde é criada e vivida onde as pessoas aprendem, trabalham, brincam e amam (Innstrand \& Christensen, 2018). Neste sentido, a PS emerge e fundamenta-se como um espaço a ser constituído por iniciativas intersetoriais, que se caracterizam pela busca de uma integração de setores da sociedade para o enfrentamento dos amplos desafios na saúde individual e coletiva (Lima \& Althaus, 2016; Azevedo et al., 2012).

Logo, a PS constitui espaço interdisciplinar para um pensar teórico-prático, tanto nos meios científicos e acadêmicos, como nas esferas profissionais (Garves et al., 2018). Tal espaço não está apenas reservado aos profissionais da saúde ou instituições públicas, mas estende-se a todos os setores e instituições organizadas da sociedade (Moraes, 2017).

A Organização Pan-Americana de Saúde (OPAS) caracteriza os locais de trabalho promotores de saúde, como uma prioridade para PS (Carvalho \& Dias, 2012). Entre os princípios fundamentais estão: característica participativa e fortalecedora; a cooperação multissetorial e multidisciplinar; a justiça social; a sustentabilidade e a completude (Casas \& Klijn, 2006). Em uma interessante revisão sistemática nominada "Promoção da saúde no local de trabalho: revisão sistemática da literatura", Carvalho e Dias (2012) além de classificar os artigos inclusos em sua pesquisa por áreas temáticas, também organizam o corpus de acordo com o foco principal das ações e intervenções de PS, em individuais, sociais e organizacionais.

O ambiente escolar visto pela dimensão organizacional se constitui como espaço promotor de saúde não apenas voltado a práticas formativas do discente, mas como lugar oportuno para ação e promoção de ambientes de trabalho saudáveis, ou seja, centradas nos professores e profissionais atuantes no espaço escolar (Innstrand \& Christensen, 2018).

Diante dessa realidade, há premente necessidade de compreender o campo da saúde do trabalhador, relacionado ao trabalho do professor, como contributo na produção de melhorias que favoreçam a qualidade de vida (QV), bem como a PS individual e coletiva dos professores, com desejáveis compreensões interdisciplinares sobre o tema (Innstrand \& Christensen, 2018; Cortez et al., 2017; Davoglio et al., 2015).

A sociedade contemporânea necessita de professores que tenham posturas fundamentadas na prática reflexiva e na implicação crítica (Perrenoud \& Thurler, 2007). Dessa forma, promover o debate de professores sobre as atuais práticas sociais, envolve considerar o assunto QV e estilo de vida (EV), uma vez que se mostram relacionados diretamente à saúde (Miranda et al., 2018; Pereira et al., 2017; Dankel et al., 2016). 
Explicita-se que o EV relacionado a saúde tem se caracterizado como conjunto de hábitos e costumes que são influenciados, modificados, fomentados ou inibidos pelo processo de socialização ao longo da vida, enquanto a QV refere-se a percepção do indivíduo sobre seu bem-estar físico, mental, psicológico e espiritual, relaciona-se à saúde, educação, e interação social com os amigos e familiares (Miranda et al., 2018; Pereira et al., 2017).

Os entrelaçamentos entre EV e QV na PS do professor ainda é comedida, pouco investigados na literatura científica. As publicações disponíveis se alinham à saúde no trabalho do professor com predomínio de publicações nas áreas de fonoaudiologia e psicologia, evidenciando o adoecimento desse grupo, apontando para a necessidade de desenvolvimento de ações de PS para o professor (Cortez et al., 2017). No mesmo sentido, parecem ainda mais escassas as investigações sobre as referidas relações entre professores de escolas técnicas (Santos et al., 2016; Alvares \& Ziviani, 2015; Mello, 2014).

Sendo assim desejáveis, estudos que avaliem indicadores que se relacionam com a saúde deste grupo. Este estudo teve por objetivo analisar o perfil de saúde de professores de uma Escola Técnica Estadual (ETEC) da grande São Paulo, implementar e divulgar no blog desta instituição um programa educativo voltado à promoção da alimentação saudável entre os professores.

\section{Metodologia}

Trata-se de estudo transversal, quantitativo e descritivo com amostra não probabilística (Pereira et al., 2018). Foram respeitadas as diretrizes da Declaração de Helsinque (WMA, 2013) e da Resolução 466/12 do Conselho Nacional de Saúde (CNS) (Brasil, 2012), com aprovação pelo Comitê de Ética em Pesquisa com parecer sob CAAE: 31132620.4.0000.5377 e autorizado pela Escola Técnica Estadual (ETEC).

As ETEC pertencem ao Centro Paula Sousa, que se configura como uma Autarquia do Governo do Estado de São Paulo, vinculada à Secretaria de Desenvolvimento Econômico, Ciência, Tecnologia e Inovação.

O convite para a participação na pesquisa foi feito por meio de carta-convite enviada ao grupo de professores já formado na plataforma Microsoft Teams. Nesse convite foram prestados esclarecimentos, em relação à pesquisa e seus objetivos, destacando-se que as avaliações que seriam realizadas não afetariam a evolução funcional do professor.

A ETEC, onde foi realizada a pesquisa possui em torno de 79 professores. Desses, 24 (30,4\%) professores não desenvolviam suas atividades na unidade sede, ou porque haviam passado a lecionar em outras ETEC ou por conta de afastamentos por motivos pessoais ou ocupacionais. Dessa forma, foram convidados a participar $55(69,6 \%)$ professores que estão em atividade na ETEC, destes, 43 aceitaram participar do estudo.

Não foram incluídos na amostra professores que não tinham aula atribuída no período da coleta de dados e que estavam afastados de suas atividades por problemas ocupacionais.

A coleta de dados foi realizada por meio da plataforma de questionários online Google Forms. Os participantes receberam o Termo de Consentimento Livre e Esclarecido e três questionários (autoaplicáveis): primeiro questionário continha as variáveis sociodemográficas e o questionário "Como está sua alimentação?”; o segundo foi sobre conhecimento em nutrição e o terceiro contemplou o Instrumento "Fantástico" de avaliação do estilo de vida e o WHOQOL-bref para avaliação da qualidade de vida.

Os dados sociodemográficos foram as variáveis: sexo (feminino ou masculino), idade (anos), estado civil (solteiro, casado, viúvo, união estável ou divorciado) profissão/ocupação, bem como informações complementares sua formação.

As informações coletadas para a classificação socioeconômica foram referentes à escolaridade do chefe da família e a presença de bens de consumo no domicílio. Os dados foram analisados de acordo com o Critério de Classificação Econômica Brasil por meio do questionário da Associação Brasileira de Empresas de Pesquisa (ABEP), o qual atribui pontos e classifica as classes socioeconômicas em A1, B1, B2, C1, C2, D-E (ABEP, 2016). 
O consumo alimentar e outros hábitos de vida foi avaliado pelo questionário do Ministério da Saúde "Como está sua alimentação?", composto por 18 questões de múltipla escolha, as perguntas contemplam aspectos do consumo alimentar contido no Guia Alimentar: Como ter uma alimentação saudável (Brasil, [s.d]).

A pontuação final do questionário é o resultado do somatório de todas as questões, que podem variar de 1 a 58 . Os que alcançaram até 28 pontos precisam tornar sua alimentação e hábitos de vida mais saudáveis; entre 29-42 pontos, os indivíduos devem ter mais atenção com a alimentação e hábitos de vida e os que tiveram 43 pontos ou mais estão no caminho para um modo de vida saudável (Brasil, [s.d]). Neste estudo para análise das três categorias relativas à qualidade do consumo alimentar e outros hábitos de vida, foram adotados os seguintes termos: "Precisa melhorar", "Atenção" e "Parabéns", respectivamente.

A Escala de Conhecimento em Nutrição permitiu avaliar os conhecimentos em nutrição e o instrumento é dividido em três partes. No questionário, as pontuações totais entre 0 e 6 indicam baixo conhecimento em nutrição; entre 7 e 10 apontam moderado conhecimento nutricional e acima de 10 revelam alto conhecimento nutricional (Scagliusi et al., 2006).

O questionário "Estilo de vida fantástico" é um instrumento genérico que foi traduzido e validado para o português do Brasil por Rodriguez-Añez et al. (2008).

O questionário é composto por 25 questões divididas em nove domínios: famílias e amigos; atividade física; nutrição; cigarros e drogas; álcool; sono, cinto de segurança, estresse e sexo seguro; tipo de comportamento; introspecção e trabalho (Rodriguez-Añez et al., 2008).

A soma dos pontos permite chegar a um escore total que classifica os indivíduos em cinco categorias de EV: "Excelente" com a pontuação entre 85 a 100 pontos, "Muito bom" para aqueles com a pontuação entre 70 a 84 pontos, "Bom" 55 a 69 pontos, "Regular" de 35 a 54 pontos e "Necessita melhorar" de 0 a 34 pontos (Rodriguez-Añez et al., 2008).

A QV foi avaliada pelo questionário WHOQOL-bref (World Health Organization Quality on Life). Instrumento utilizado para aferir quantitativamente a QV (Fleck et al., 2000).

A versão breve possui 26 perguntas, com cinco opções de resposta: 24 facetas (uma pergunta para cada faceta) a respeito de quatro domínios da vida: 1. Físico, 2. Psicológico, 3. Relações sociais e 4. Meio ambiente; e duas questões separadas de caráter mais geral, que questionam diretamente a avaliação de QV e a satisfação pessoal com o estado de saúde. É autoexplicativo e as respostas referem-se às duas últimas semanas (Fleck et al., 2000).

O Programa educativo foi elaborado e implementado para os professores desta ETEC com objetivo de discutir e promover uma reflexão sobre alimentação saudável e foi realizado em dois encontros via zoom. O material usado nesse programa foi organizado em uma produção técnica divulgada no blog da ETEC para que todos tivessem acesso à informação (http://etecdebarueri.blogspot.com/2020/10/alimentacao-saudavel-e-equilibrada.html?m=1).

Os encontros tiveram duração de duas horas cada um, foram realizados em horários livres de aulas e com a participação de uma nutricionista, que contribuiu com conteúdo técnico e explicação das informações relacionadas à nutrição. Foram expostos aos professores os assuntos que seriam discutidos durante os dois encontros. Eles puderam sugerir assuntos de seus interesses para a discussão no segundo encontro, que foram o número e tamanho das porções de alimentos que compõem uma dieta de $2000 \mathrm{kcal}$, recomendada para população adulta (BRASIL, 2008).

O primeiro encontro teve como tema "Falando sobre alimentação e nutrição", neste promoveu-se uma reflexão sobre o panorama nutricional no Brasil, mostrando dados sobre o excesso de peso, consumo alimentar e prática de atividade física da população brasileira. Optou-se por relembrar os grupos de alimentos de acordo com o grau de processamento: in natura, processados e ultraprocessados, demonstrando os alimentos que fazem parte de cada grupo, seus efeitos na saúde e como devem ser inseridos na rotina alimentar.

O segundo encontro teve como tema "Alimentação equilibrada", foram discutidos os aspectos que influenciam os hábitos alimentares, os grupos de alimentos para compor uma dieta de $2000 \mathrm{kcal}$, número de porções por grupo alimentar, 
exemplos de alimentos e suas porções em medidas caseiras e por fim foram apresentados e discutidos os "Dez passos para alimentação saudável” (Brasil, 2014).

As análises do presente estudo foram conduzidas no Statistical Package for the Social Sciences (SPSS) versão 24 para Windows. O nível de significância estabelecido foi de $\mathrm{p}<0,05$ para se rejeitar a hipótese nula.

Inicialmente foram realizadas análises descritivas de todas as variáveis de estudo, testando, previamente, sua normalidade ou não por meio do Teste de D'Agostino-Pearson. As diferentes prevalências encontradas foram avaliadas por meio do teste do qui-quadrado.

As associações bivariadas foram determinadas por meio do coeficiente de correlação (r) de Pearson (variáveis numéricas) ou Spearman (variáveis categóricas). Os valores foram interpretados segundo proposto por Cohen e adaptado por Mukaka (2012), como segue: muito fraca: $r<0,20$, fraca: $r<0,40$, moderada: $r<0,60$, forte: $r<0,80$, e muito forte: $r \geq 0,80$.

Determinou-se o alfa de Cronbach para avaliar quão uniformemente os itens dos questionários contribuíam para a soma não ponderada de cada instrumento para a amostra estudada. Valores do alfa de Cronbach inferiores 0,50 foram considerados abaixo do aceitável, e os iguais ou superiores a 0,50 como aceitáveis para uma amostra inferior a 50 indivíduos (Maroco \& Garcia-Marques, 2006).

\section{Resultados}

Foram avaliados 43 indivíduos de 31 a 62 anos $(46,1 \pm 8,0$ anos) sendo a maioria do sexo feminino $(51,2 \%)$. A estatura média \pm desvio-padrão foi de 170,6 \pm 9,2 cm (153,0 a 187,0 cm), peso corporal médio de 83,5 $\pm 19,2 \mathrm{~kg}(52,0$ a 153,5 kg) e IMC médio de $28,5 \pm 5,2 \mathrm{~kg} / \mathrm{m} 2(19,3$ a 48,6 kg/m2). O estado nutricional de acordo com o IMC indicou predominância de excesso de peso $(69,8 \%)$, sendo $41,9 \%$ ( $n=18)$ com sobrepeso e $27,9 \%$ ( $n=12)$ com obesidade.

A classe socioeconômica mostrou predomínio da classe B com 62,8\% (n=27). Em relação à formação, 86,1\% (n=37) tinham especialização, 9,3\% (n=4) mestrado e 4,7\% (n=2) doutorado, como apresentado na Tabela 1. 
Tabela 1 - Caracterização sociodemográfica, antropométrica e de formação profissional de professores (n=43) de uma ETEC na grande São Paulo, 2020.

\begin{tabular}{lc}
\hline Variáveis & $\mathbf{n}(\boldsymbol{\%})$ \\
\hline Sexo & $22(51,2)$ \\
Feminino & $21(48,8)$ \\
Masculino & \\
Estado civil & $32(74,4)$ \\
Com companheiro/a & $11(25,6)$ \\
Sem companheiro/a & \\
Classificação & $11(25,6)$ \\
Eutrófico & $18(41,9)$ \\
Sobrepeso & $12(27,9)$ \\
Obesidade & \\
Classe socioeconômica & $08(18,6)$ \\
A & $27(62,8)$ \\
B & $08(18,6)$ \\
C & \\
Formação profissional & \\
Graduação & $13(30,2)$ \\
Licenciaturas & $6(14,0)$ \\
Tecnológicas & $10(23,6)$ \\
Ciências Sociais Aplicadas & $14(32,6)$ \\
Ciências da Saúde & \\
Pós-graduação & $37(86,1)$ \\
Especialização (Pós-graduação lato sensu e MBA) & $04(9,3)$ \\
Mestrado & $02(4,7)$ \\
Doutorado &
\end{tabular}

Fonte: Dados da pesquisa (2020).

Com relação à QV, apenas 6\% dos avaliados exibiu QV regular, 76\% boa QV e 18\% muito boa QV. Entre os domínios da QV, o meio ambiente teve a menor pontuação, 63,6. Em relação ao EV, nenhum participante exibiu EV classificado como "regular" ou "ruim", 60,4\% exibiu EV classificado como "bom" e 39,5\% EV classificado como "muito bom", na Tabela 2 constam as informações referentes às médias e desvio-padrão do estilo e qualidade de vida.

Tabela 2 - Caracterização do estilo e qualidade de vida de professores $(n=32)$ de uma ETEC na grande São Paulo, 2020.

\begin{tabular}{lc}
\hline Variáveis & Média \pm DP \\
\hline Estilo de vida & $67,3 \pm 6,6$ \\
Escore total & \\
Qualidade de vida & $71,4 \pm 8,2$ \\
Escore total & \\
Domínios da Qualidade de vida & $73,7 \pm 9,8$ \\
Domínio físico & $74,2 \pm 8,9$ \\
Domínio psicológico & $74,2 \pm 14,2$ \\
Domínio relações sociais & $63,6 \pm 10,2$ \\
Domínio meio ambiente & \\
\hline
\end{tabular}

Fonte: Dados da pesquisa (2020).

Quanto ao consumo alimentar e outros hábitos de vida, 21\% foram classificados em "precisa melhorar", 79\% em “atenção" e nenhum professor apresentou classificação em "parabéns", ou seja, pontuação máxima nessa variável. A avaliação do conhecimento em nutrição indicou que $25,6 \%$ exibia baixo conhecimento em nutrição, $65,1 \%$ moderado e $9,3 \%$ alto 
conhecimento, como indicado na Tabela 3.

Tabela 3 - Caracterização do consumo alimentar e outros hábitos de vida e conhecimento em nutrição de professores de uma ETEC na grande São Paulo, 2020.

\begin{tabular}{lc}
\hline Variáveis & $\mathbf{n}(\%)$ \\
\hline Consumo alimentar e outros hábitos de vida & \\
Precisa melhorar & $09(20,9)$ \\
Atenção & $34(79,0)$ \\
Conhecimento em nutrição & \\
Baixo & $11(25,6)$ \\
Moderado & $28(65,1)$ \\
Alto & $04(9,3)$ \\
\hline
\end{tabular}

Fonte: Dados da pesquisa (2020).

O consumo alimentar e outros hábitos de vida também foram avaliados em comportamento adequado e inadequado em cada questão do questionário. Verificou-se prevalências de comportamento inadequado nas questões 1, 2, 4, 7, 8, 9, 14, 16, 17, ou seja, houve inadequação em metade dos aspectos avaliados, incluindo a prática de atividade física, o consumo de frutas, legumes e verduras, alimentos fontes de carboidratos, leite e derivados, bebidas alcoólicas, consumo de peixe e número de refeições como demonstrado na Tabela 4.

Tabela 4 - Consumo alimentar e outros hábitos de vida por questão, de professores de uma ETEC na grande São Paulo, 2020.

\begin{tabular}{|c|c|c|}
\hline Questões & $\begin{array}{l}\text { Comportamento } \\
\text { Adequado } \\
\text { n (\%) }\end{array}$ & $\begin{array}{l}\text { Comportament } \\
\text { o Inadequado } \\
\text { n }(\%)\end{array}$ \\
\hline $\begin{array}{l}\text { 1. Qual é, em média, a quantidade de frutas (unidade/ } \\
\text { fatia/pedaço/copo de suco natural) que você come por dia? }\end{array}$ & $10(23,3 \%)$ & $33(76,7 \%)$ \\
\hline $\begin{array}{l}\text { 2. Qual é, em média, a quantidade de legumes e verduras que você } \\
\text { come por dia? }\end{array}$ & $8(18,6 \%)$ & $35(81,4 \%)$ \\
\hline $\begin{array}{l}\text { 3. Qual é, em média, a quantidade que você come dos seguintes } \\
\text { alimentos: feijão de qualquer tipo ou cor, lentilha, ervilha, grão-de-bico, } \\
\text { soja, fava, sementes ou castanhas? }\end{array}$ & $25(58,1 \%)$ & $18(41,9 \%)$ \\
\hline $\begin{array}{l}\text { 4. Qual a quantidade, em média, que você consome por dia dos } \\
\text { alimentos fontes de carboidratos como pães, massas, biscoitos, bolo entre } \\
\text { outros que estão listados? }\end{array}$ & $5(11,6 \%)$ & $38(88,4 \%)$ \\
\hline $\begin{array}{l}\text { 5. Qual é, em média, a quantidade de carnes (gado, porco, aves, } \\
\text { peixes e outras) ou ovos que você come por dia? }\end{array}$ & $34(79,1 \%)$ & $9(20,9 \%)$ \\
\hline $\begin{array}{l}\text { 6. Você costuma tirar a gordura aparente das carnes, a pele do frango } \\
\text { ou outro tipo de ave? }\end{array}$ & $30(69,8 \%)$ & $13(30,2 \%)$ \\
\hline Você costuma comer peixes com qual frequência? & $6(14,0 \%)$ & $37(86,0 \%)$ \\
\hline $\begin{array}{l}\text { 8. Qual é, em média, a quantidade de leite e seus derivados (iogurtes, } \\
\text { bebidas lácteas, coalhada, requeijão, queijos e outros) que você come por } \\
\text { dia? }\end{array}$ & $5(11,6 \%)$ & $38(88,4 \%)$ \\
\hline Que tipo de leite e seus derivados você habitualmente consome? & $15(34,9 \%)$ & $28(65,1 \%)$ \\
\hline $\begin{array}{l}\text { 10. Pense nos seguintes alimentos: frituras, salgadinhos fritos ou em } \\
\text { pacotes, carnes salgadas, hambúrgueres, presuntos e embutidos (salsicha, }\end{array}$ & $30(69,8 \%)$ & $13(30,2 \%)$ \\
\hline
\end{tabular}


mortadela, salame, linguiça e outros). Você costuma comer qualquer um deles com que frequência?

11. Pense nos seguintes alimentos: doces de qualquer tipo, bolos $27(62,8 \%)$ $16(37,2 \%)$ recheados com cobertura, biscoitos doces, refrigerantes e sucos industrializados. Você costuma comer qualquer um deles com que frequência?

12. Qual tipo de gordura é mais usado na sua casa para cozinhar os alimentos?

13. Você costuma colocar mais sal nos alimentos quando já servidos em seu prato?

14. Pense na sua rotina semanal: quantas refeições você costuma fazer habitualmente no dia?

15. Quantos copos de água você bebe por dia? Inclua no seu cálculo sucos de frutas naturais ou chás (exceto café, chá preto e chá mate).

16. Você costuma consumir bebidas alcoólicas (uísque, cachaça, vinho, cerveja, conhaque etc.) com qual frequência?

17. Você faz atividade física regular, isto é, pelo menos 30 minutos por dia, todos os dias da semana, durante o seu tempo livre?

18. Você costuma ler a informação nutricional que está presente no rótulo de alimentos industrializados antes de comprá-los?

$39(90,7 \%)$

$4(9,3 \%)$

$42(97,7 \%)$

$11(25,6 \%)$

$26(60,5 \%)$

$11(25,6 \%)$

$7(16,3 \%)$

$36(83,7 \%)$

$27(62,8 \%)$

Fonte: Dados da pesquisa (2020).

Não foram identificadas correlações significantes entre consumo alimentar e conhecimento nutricional com as demais variáveis. A idade se correlacionou positivamente ao domínio psicológico da $\mathrm{QV}(\mathrm{r}=0,36, \mathrm{p}=0,03)$ e com o escore da classificação socioeconômica $(r=0,29, \mathrm{p}=0,05)$. Isso quer dizer que quanto maior a idade melhor o escore no domínio psicológico da QV e melhor classificação socioeconômica. O domínio social da QV correlacionou-se inversamente com escore da classificação socioeconômica $(r=-0,34, p=0,05)$. Ou seja, quanto menor o escore da classificação socioeconômica melhor o escore no domínio social da QV.

O escore total de avaliação do EV correlacionou-se positivamente com o escore total (r=0,39, p=0,02), com o domínio psicológico $(r=0,35, \mathrm{p}=0,04)$ e com o domínio social $(\mathrm{r}=0,34, \mathrm{p}=0,05)$ da QV. Em outras palavras, quanto maior o escore do EV maior a QV geral e nos domínios psicológico e social. Ressalta-se que essas correlações foram consideradas fracas.

Quanto a participação no Programa educativo dos 43 professores que responderam aos questionários, apenas 4 participaram dos encontros realizados por zoom para a discussão e promoção da alimentação saudável.

\section{Discussão}

O objetivo principal deste trabalho foi traçar o perfil de saúde de professores de uma escola técnica estadual. Destacase o predomínio de adultos, com excesso de peso, classe socioeconômica B, com formação em nível de especialização e que viviam com companheiro. Houve prevalência de EV e QV classificados como "bom" e a maioria necessitava implementar melhorias no consumo alimentar e outros hábitos de vida.

Comparando-se a realidade internacional, o Brasil ocupa um dos últimos lugares na educação técnica, onde o índice é de apenas 8\%, enquanto nos países membros da Organização para Cooperação e Desenvolvimento Econômico (OCDE) têm-se, em média, 43\% dos estudantes entre 15 e 19 anos matriculados em cursos técnicos (Moraes \& Albuquerque, 2019). Os dados estatísticos sobre a educação profissional e tecnológica (EPT), de nível médio ou superior no Brasil, ainda são limitados, mesmo 
diante da sua relevância social (Moraes \& Albuquerque, 2019).

Neste sentido a figura do professor do ensino técnico profissionalizante tem como característica, o processo contínuo de formação que surge das suas práticas pedagógicas e articula-se com o contexto social e institucional nos quais estão inseridos. Dessa forma, torna-se necessário o conhecimento dos princípios institucionais onde atua, da prática em sala de aula e dos motivos de suas escolhas pessoais e profissionais, cujas consequências políticas influenciam na vida dos estudantes, ultrapassando o mero treinamento e desenvolvimento de competências laborais (Carneiro \& Cavalcante, 2018).

Explicita-se que a presente pesquisa foi trazida a efeito em instituição técnica de nível médio pública estadual, porém instituições de educação tecnológica, podem ser de natureza pública (estadual ou federal) ou privada, e por vezes congregam a educação profissionalizante mediada via cursos de curta duração, além da educação técnica de nível médio e superior (Santos et al., 2016).

Assim é usual a comparação de variáveis do perfil de professores de escolas técnicas por natureza da oferta (público/privada), por natureza do ensino (técnico e não técnico), por nível de ensino (médio ou superior), por classe ocupacional (docência), ainda que nem sempre estejam relatadas claramente estas características (Silva, 2019; Soares \& Henig, 2019; Conte \& Paula, 2016a; 2016b; Carvalho \& Souza, 2014).

Em relação ao sexo, de maneira geral existe certo equilíbrio nos estudos disponíveis de perfis de professores de escolas técnicas estaduais de nível médio, ora apresentando leve prevalência masculina (Santos et al., 2016; Lima et al., 2017) pra feminina (Silva, 2019). Semelhante ao presente estudo em que houve proporção feminina levemente superior (51,2\%).

Em relação a faixa etária, os estudos com professores do ensino médio e técnico de nível médio é prevalente idade acima dos 30 anos, e em geral na meia idade (35 a 65 anos), e com idade média por volta dos 40 anos (Conte \& Paula, 2016b; Soares \& Henig, 2019; Vedovato \& Monteiro, 2008), semelhante ao presente estudo.

Quanto a situação conjugal, uma ampla pesquisa ocupacional realizada com professores da educação básica no Brasil, incluindo o ensino fundamental e médio, e o público e o privado, com maioria da amostra composta por indivíduos da região sudeste (40,5\%), indicou prevalente autodeclaração de viver com companheiro (59,2\%) (Assunção \& Abreu, 2019). No presente estudo houve proporção superior de professores com autorrelato conjugal de que possuem companheiro $(74,4 \%)$. Essa diferença talvez possa estar ligada a características socioculturais onde se realizaram os estudos.

Em relação à classe socioeconômica no presente estudo houve predominância de professores classificados na classe B (62,8\%), semelhante a investigação de Silva (2019) sobre QV e prática de atividades físicas de professores de ensino médio de instituições escolares estaduais técnica e de referência em Brasília, Distrito Federal, onde também houve prevalência de professores classificados na classe B $(64,7 \%)$.

Em relação à formação acadêmica Lima et al. (2017) na sua pesquisa no estado de São Paulo com professores do ensino médio (propedêutico) integrado ao técnico (profissional) em escolas técnicas estaduais ligadas ao Centro Estadual de Educação Tecnológica Paula Souza (CEETEPS), verificaram maior proporção de especialistas $(53,1 \%)$, e nenhum professor com nível de doutorado. No presente estudo, na ETEC pesquisada, também ligada ao CEETEPS, houve proporção ainda mais alta de professores especialistas $(86,1 \%)$, bem como a presença de mestres $(9,3 \%)$ e doutores $(4,7 \%)$.

Quanto ao consumo alimentar dos participantes no presente estudo, foram observados alguns hábitos alimentares que divergem das recomendações e orientações expressas no Guia Alimentar para a População Brasileira (Brasil, 2008), com autorrelato de inadequação no consumo frutas, legumes, peixes, leite, bebidas alcoólicas e número de refeições por dia. Diante disso, recomenda-se alimentação baseada em uma grande variedade de alimentos in natura ou minimamente processados; que ingredientes culinários processados sejam utilizados em pequena quantidade para transformar alimentos do primeiro grupo em preparações culinárias. Os alimentos processados, também devem ser utilizados em pequenas quantidades e como parte ou para acompanhar preparações culinárias; e, finalmente, que o consumo de alimentos ultraprocessados seja evitado (Brasil, 2014). 
Ressalta-se que a amostra estudada no presente estudo segue a linha de resultados expressas em grandes inquéritos populacionais, como a Pesquisa de Orçamentos Familiares (POF) 2017-2018, que evidencia evolução da disponibilidade domiciliar de alimentos no Brasil, baseada na comparação de resultados das POF anteriores (2002-2003, 2008-2009 e 20172018), indicando que alimentos in natura ou minimamente processados e ingredientes culinários processados vêm perdendo espaço para alimentos processados e, sobretudo, para alimentos ultraprocessados (IBGE, 2020). De acordo com o Sistema de vigilância de fatores de risco e proteção para doenças crônicas por inquérito telefônico (Vigitel) 2019, no estado de São Paulo apenas 34,3\% da população consome frutas e hortaliças em cinco ou mais dias da semana (Brasil, 2020)

No presente estudo somente $23,3 \%$ consomem frutas diariamente, e em relação a legumes e verduras apenas 18,6\% consomem adequadamente, valores menores que os resultados do Vigitel 2019 (Brasil, 2020). Outro ponto que merece ser mencionado é a pouca influência da escolaridade dos professores deste estudo no seu consumo de frutas, verduras e legumes, já que segundo Figueiredo (2006) a escolaridade é um dos determinantes positivos para o consumo desses alimentos. O Vigitel 2019 verificou aumento no consumo de frutas, verduras e legumes entre outros aspectos positivos do consumo alimentar com o aumento da escolaridade (Brasil, 2020).

A POF 2017-2018 descreve que na população brasileira as fontes de carboidratos são o arroz (15,6\%), feijão (4,3\%), o macarrão $(2,7 \%)$, pães $(6,7 \%)$, bolos e tortas doces $(1,5 \%)$, farinha de milho, fubá e outras $(2 \%)$, farinha de mandioca $(1,9 \%)$, farinha de trigo $(1,8 \%)$ e as raízes e tubérculos $(1,2 \%)$. Já no presente estudo, apesar de não ser avaliado individualmente as fontes de carboidratos, houve autorrelato de $84,4 \%$ classificados como "inadequado" pelo baixo consumo desses alimentos fontes de carboidratos complexos.

Em relação ao consumo de peixes, $86,0 \%$ da nossa amostra apresentou consumo inadequado, abaixo do recomendado. A POF 2017-2018 indica consumo desse alimento por apenas 0,5\% da população brasileira. O que pode estar relacionado a diferenças socioculturais no território, bem como aos elevados custos. Quanto ao tipo e quantidade do consumo de leite e seus derivados, a amostra deste estudo apresentou consumo considerado inadequado, abaixo do recomendado para a população adulta (Brasil, 2008). A POF 2017-2018 explicita que apenas 5\% da população consome leite, não especificando qual o tipo de leite (IBGE, 2020).

O consumo de bebidas alcoólicas (fermentadas e destiladas) na presente pesquisa também foi considerado inadequado (acima do limite estabelecido) para 74,4\% dos professores. A POF apresenta que 0,7\% da população consome bebida alcoólica fermentada e 0,2 bebida alcoólica destilada (IBGE, 2020). E o Vigitel 2019 teve um percentual de consumo de 16,3\% da amostra de adultos ( $\geq 18$ anos) pesquisada em São Paulo (Brasil, 2020). Outro dado que merece destaque é que 74,4\% da amostra do presente estudo não realiza número adequado de refeições por dia, considerado entre 5 ou 6 refeições diárias (Brasil, [s.d]).

Em uma pesquisa realizada com professores do ensino médio técnico sobre educação alimentar e nutricional numa abordagem interdisciplinar, verificou-se que os professores consideram os benefícios dessas medidas como importantes para a atuação em sala de aula. Esses conteúdos também auxiliam nas questões relacionadas à saúde e QV reforçando a orientação da Base Nacional Comum Curricular (BNCC) em abordar de forma conjunta às outras disciplinas assuntos relativos à alimentação saudável. No entanto, apesar de o estudo não fazer inferências sobre os padrões de conhecimento ou estado nutricional dos participantes, reafirma que há dificuldade por parte dos professores em lidar com o assunto de forma transdisciplinar (Mercante \& Messias, 2019).

Salienta-se que no estudo de Rocha et al. (2015) sobre a prevalência e os fatores sociodemográficos, ocupacionais e hábitos de vida associados ao sobrepeso entre professores da educação básica da rede estadual de um município da Bahia, apresentou média de IMC de $24,93 \mathrm{~kg} / \mathrm{m} 2 \pm$ 4,38 e prevalência de excesso de peso de 47,2\%.

$\mathrm{O}$ aumento da prevalência de excesso de peso na população brasileira e sua caracterização como problema de saúde pública é demonstrada em estudos como o Vigitel 2019 que mostrou elevada prevalência geral de excesso de peso (55,4\%), 
ressaltando o papel da escolaridade para diminuição da incidência (Brasil, 2020). A média do IMC da presente pesquisa foi 28,5 $\mathrm{kg} / \mathrm{m} 2 \pm 5,2$ e predominância de excesso de peso $(69,8 \%)$ superior a proporção dos estudos citados. Esta diferença pode estar relacionada a especificidade da amostra, ao recorte temporal da pesquisa, ou mesmo a padrões alimentares socioculturais diferentes pré-estabelecidos no território.

No presente estudo a avaliação da QV foi considerada boa, tendo o menor escore na dimensão meio ambiente, semelhante a outros estudos com professores de escolas técnicas estaduais e federais de nível médio, bem como em estudos com professores da educação básica, tanto no nível fundamental como médio (Silva, 2019; Brum, 2018; Lopes et al., 2018b; Tabeleão et al., 2011; Penteado \& Pereira, 2007).

Os estudos disponíveis consultados evidenciam os escores médio do domínio meio ambiente da QV, como o mais prejudicado, como já mencionado. O domínio meio ambiente engloba questões como a falta, a insuficiência, inadequação ou insatisfação com o transporte, o lazer, a proteção, questões financeiras, serviços de saúde e ambiente de trabalho (Penteado \& Pereira, 2007). Este cenário convergente em estudos com diferentes esferas e níveis de atuação de professores, evidencia a precariedade e desvalorização da função ocupacional, denunciada como "mal-estar docente" (Zaragoza, 1999).

Ainda no que diz respeito a QV em análise de associação com as outras variáveis do presente estudo, foi identificada correlação fraca, mas significante, indicando que quanto maior a idade, maior a classificação socioeconômica e maiores escores médios no domínio psicológico da QV, e quanto menor o escore médio da classificação socioeconômica maior o escore médio no domínio social da QV. Salienta-se que a ABEP (2016) descreve como observações importantes, que seu questionário define grandes classes por poder aquisitivo, entretanto, como qualquer outro critério, não consegue satisfazer todos os usuários em todas as circunstâncias. E sublinha-se que o domínio social avalia relações sociais dentre elas relações pessoais, suporte (apoio) social e atividade sexual, corroborando com dados mais subjetivos e qualitativos das relações humanas.

Logo pressupõe-se que a associação negativa entre domínio social da QV e a classificação socioeconômica no presente estudo, a princípio incompreendida, pode ser produto ou fator de confusão da relação de outras variáveis do estudo (Lima-Costa \& Barreto, 2003), ou ainda pode estar relacionada a extensas jornadas de trabalho, em função da manutenção do padrão econômico desejado, desfavorecendo importantes aspectos do convívio social (Araujo et al., 2019; Silva et al., 2019).

Houve ainda no presente estudo associação positiva em que, quanto maior o escore médio de EV maior o escore médio geral e nos domínios psicológico e social da QV, além de prevalência de EV classificado como "bom". Ressalta-se que essas correlações são corroboradas por outros estudos com diferentes populações, evidenciando a associação positiva entre componentes do EV e QV (Miranda et al., 2018; Pereira et al., 2017), porém parecem escassos estudos que abordem essa associação entre professores da educação profissional técnica de nível médio, limitando nossa discussão.

Sobre a prática de atividade física verificou-se comportamento inadequado (comportamento sedentário) em 83,7\% dos participantes no presente estudo, estando nossos números aquém dos dados do Vigitel 2019 onde 34,6\% dos pesquisados praticam atividade física no estado de São Paulo (Brasil, 2020).

Entre as limitações do estudo observa-se ter sido realizado em uma única unidade escolar, limitando nossas conclusões. Entre os pontos fortes do trabalho destaca-se a investigação das relações entre estado nutricional, EV e QV de professores de uma escola técnica estadual de nível médio, favorecendo um melhor panorama da saúde desta população no território e ambiente escolar, uma vez que a literatura carece dessas informações. Dessa forma, expondo informações sobre o EV e a QV de professores atuantes na educação profissionalizante, políticas de promoção da saúde poderão ser implementadas para um grupo de trabalhadores que são indispensáveis para a formação de uma sociedade justa, igualitária e saudável. 


\section{Conclusão}

Os professores estudados eram adultos, com excesso de peso, predomínio da classe socioeconômica B, necessitam melhorar o consumo alimentar e outros hábitos de vida, tem conhecimento moderado em nutrição e apresentam QV e EV bons. Houve correlações positivas e fracas, mas significantes, entre a idade, a classificação socioeconômica e os escores médios no domínio psicológico da QV; entre o EV e QV geral e nos domínios psicológico e social; assim como correlações fracas e negativas entre a classificação socioeconômica e o domínio social da QV.

Esses dados explicitam a necessidade de iniciativas que favoreçam o espaço escolar como ambiente promotor da saúde do professor, como mecanismo de sustentabilidade social e organizacional, tais como o programa educativo voltado a promoção da alimentação saudável, realizado e publicado no blog institucional desta ETEC, onde o presente estudo foi realizado.

Neste sentido são desejáveis propostas integradoras de pesquisas e ações intersetoriais, para a implementação de práticas contextualizadas no ambiente escolar que envolvam toda a comunidade escolar e arredores visando a promoção da saúde de maneira efetiva.

\section{Referências}

Associação Brasileira de Empresas de Pesquisa. (2016). Critério Brasil: Critério de Classificação Econômica. http://www.abep.org/criterio-brasil.

Alvares, M. S. \& Ziviani, F. (2015). Qualidade de vida no trabalho: um estudo de caso de docentes e funcionários na ETFG-BH. FACEF PesquisaDesenvolvimento e Gestão, 18(1), 95-127. https://doi.org/10.5102/un.gti.v6i1.3875

Araújo, T. M., Pinho, P. S. \& Masson, M. L. V., (2019). Trabalho e saúde de professoras e professores no Brasil: reflexões sobre trajetórias das investigações, avanços e desafios. Cadernos Saúde Pública, 35(sup 1:e00087318), 01-14. https://doi.org/10.1590/0102-311X00087318.

Assunção, A. Á. \& Abreu, M. N. S. (2019). Pressão laboral, saúde e condições de trabalho dos professores da Educação Básica no Brasil. Cadernos de Saúde Pública, 35(sup 1:e00169517), 01-16. https://doi.org/10.1590/0102-311X00169517.

Azevedo, E., Pelicioni, M. C. F. \& Westphal, M. F. (2012). Práticas intersetoriais nas políticas públicas de promoção de saúde. Physis: Revista de Saúde Coletiva. 22(4), 1333-1356. https://doi.org/10.1590/S0103-73312012000400005

Brasil. [s.d.]. Ministério da Saúde. Guia alimentar: como ter uma alimentação saudável. https://bvsms.saude.gov.br/bvs/publicacoes/gui a_alimentar_alimentacao_saudavel_1edicao.pdf.

Brasil. (2013). Ministério da Saúde. Conselho Nacional de Saúde. Resolução $n^{\circ} 466$, de 12 de dezembro de 2012. Aprova as diretrizes e normas regulamentadoras de pesquisas envolvendo seres humanos. [Internet]. Diário Oficial da União. N 12, seção 1, p. 59, Brasília. http://bit.ly/1mTMIS3.

Brasil. (2014). Ministério da Saúde. Secretaria de Atenção à Saúde. Departamento de Atenção Básica. Guia alimentar para a população brasileira. 2. ed. Brasília, DF, 2014. https://bvsms.saude.gov.br/bvs/publicacoes/guia_alimentar_populacao_brasileira_2ed.pdf.

Brasil. (2008). Ministério da Saúde.Secretaria de Atenção à Saúde. Departamento de Atenção Básica. Guia alimentar para a população brasileira: promovendo a alimentação saudável. Brasília: 210p. http://bvsms.saude.gov.br/bvs/publicacoes/guia_alimentar_populacao_brasileira_2008.pdf.

Brasil. Ministério da Saúde. (2020) Secretaria de Vigilância em Saúde. Departamento de Análise em Saúde e Vigilância de Doenças Não Transmissíveis. Vigitel Brasil 2019: vigilância de fatores de risco e proteção para doenças crônicas por inquérito telefônico. Brasília, DF, 2020. http://bvsms.saude.gov.br/bvs/publicacoes/vigitel_brasil_2019_vigilancia_fatores_risco.pdf.

Brum, E. M. (2018). Capacidade para o trabalho e qualidade de vida: avaliação do quadro funcional em instituição de educação profissional - região oeste de Santa Catarina. 93f. Dissertação (Mestrado) - Universidade Tecnológica Federal do Paraná, Pato Branco, Paraná.

Carneiro, I. M. S. P. \& Cavalcante, M. M. D. (2018). A produção acadêmica da formação de professores na Educação Profissional. HOLOS. 34(3). 201-227. https://doi.org/10.15628/holos.2018.5993.

Carvalho, A. F. S. \& Dias, E. C. (2012). Promoção da saúde no local de trabalho: revisão sistemática da literatura. Revista Brasileira em Promoção da Saúde, 25(1). 116-126. https://doi.org/10.5020/2219.

Carvalho, O. F. \& Souza, F. H. M. (2014). Formação do docente da educação profissional e tecnológica no Brasil: um diálogo com as faculdades de educação e o curso de Pedagogia. Educação \& Sociedade, 35(128), 883-908. https://doi.org/10.1590/ES0101-73302014124974.

Casas, S. B. \& Klijn, T. P. (2006). Promoción de la salud y su entorno laboral saludable. Revista Latino-Americana de Enfermagem, 14(1),136-41. https://doi.org/10.1590/S0104-11692006000100019.

Conte, M. B. F. \& Paula, M. A. B. (2016a). A docência e o ensino técnico. Educação em Debate, 38(72), 20-32. http://www.repositorio.ufc.br/handle/riufc/23139.

Conte, M. B. F. \& Paula, M. A. B. (2016b). Quem é o docente do ensino técnico. Revista Científica on-line-Tecnologia, Gestão e Humanismo, 6(2), 77-92. 
Cortez, P. A., Souza, M. V. R., Amaral, L. O. \& Silva, L. C. A. (2017). A saúde docente no trabalho: apontamentos a partir da literatura recente. Cadernos Saúde Coletiva, 25(1), 113-122. https://doi.org/10.1590/1414-462x201700010001

Dankel, S., Leke, J. \& Loprinzi, P. (2016). Physical activity and diet on quality of life and mortality: The importance of meeting one specific or both behaviors. Int J Cardiol, 202(3), 328-30. https://doi.org/10.1016/j.ijcard.2015.09.019.

Davoglio, T. R., Lettnin, C. C. \& Baldissera, C. G. (2015) . Avaliação da qualidade de vida em docentes brasileiros: uma revisão sistemática. Pro-Posições, 26(3), 145-166. https://doi.org/10.1590/0103-7307201507807.

Figueiredo, I. C. R. (2006). Determinantes do consumo de frutas, legumes e verduras em adultos residentes no município de São Paulo. Dissertação de Mestrado, Faculdade de Saúde Pública da Universidade de São Paulo, São Paulo.

Fleck, M. P., Louzada, S., Xavier, M., Chachamovich, E., Vieira, G., Santos, L. \& Pinzon, V. (2000). Aplicação da versão em português do instrumento abreviado da qualidade de vida "WHOQOL-bref'. Revista de Saúde Pública, 34(2), 178-83.http://dx.doi.org/10.1590/S0034-89102000000200012.

Garves, W., Souza, A. C., Sánchez, F. R. R., Carvalho, M. M. G. \& Moraes, M. C. L., Zukowsky-Tavares, C. (2018). Promoção da saúde na gestão de pessoas: experiência no contexto da gestão pública. Revista Interdisciplinar de Promoção da Saúde. 1(2), 112-118. http://dx.doi.org/10.17058/rips.v1i2.11764.

Instituto Brasileiro de Geografia e Estatística - IBGE. (2020). Pesquisa de orçamentos familiares 2017-2018: avaliação nutricional da disponibilidade domiciliar de alimentos no Brasil. Coordenação de Trabalho e Rendimento. Rio de Janeiro, IBGE, 2020.

Innstrand, S. T. \& Christensen, M. C. (2018). Healthy Universities. The development and implementation of a holistic health promotion intervention programme especially adapted for staff working in the higher educational sector: the ARK study. Global health promotion, 27(1), 68-76. https://doi.org/10.1177/1757975918786877.

Lima, A. \& Althaus, D. (2016). Formação docente continuada, desenvolvimento de práticas pedagógicas em sala de aula e promoção da saúde do professor: relações necessárias. Revista Brasileira de Estudos Pedagógicos, 97(245), 97-116. https://doi.org/10.1590/S2176-6681/366113867.

Lima, R. S., Pereira, S. P. \& Kanaane, R. (2017). A construção da identidade do professor no contexto do ensino técnico integrado ao médio. Boletim Academia Paulista de Psicologia, 37(93), 322-352.

Lima-Costa, M. F. \& Barreto, S. M.(2003). Tipos de estudos epidemiológicos: conceitos básicos e aplicações na área do envelhecimento. Epidemiologia e serviços de saúde, 12(4), 189-201.

Lopes, M. C. L. A., Fagundes, S. N., Mousinho, K. C., Correia, M. G. C., Ribeiro, C. M. B. \& Vanderlei, A. D. (2018b). Fatores associados à saúde vocal e a qualidade de vida em professores. Revista CEFAC, 20(4), 515-531. https://doi.org/10.1590/1982-021620182048417.

Maroco, J. \& Garcia-Marques, T. (2006). Qual a fiabilidade do alfa de Cronbach? Questões antigas e soluções modernas? Laboratório de psicologia, 4(1), 6590. http://hdl.handle.net/10400.12/133.

Mello, R. S. J. A importância da qualidade de vida no trabalho: o caso de uma ETEC. 2014. Monografia (Especialização) - Pós Graduação em Gestão Pública, Universidade Tecnológica Federal do Paraná, Pato Branco, Paraná, 2014.

Mercante, S. C. \& Messias, C. M. B. O. (2019). A visão de docentes do ensino médio técnico sobre a educação alimentar e nutricional numa abordagem interdisciplinar. In: FERREIRA, G. R. (Org.). Educação: políticas, estrutura e organização 3. Ponta Grossa: Atena Editora.

Miranda, M. K. V., Porto, E. F. \& Souza, A. C. (2018). Influência do estilo de vida e qualidade de vida nos exames de sangue. Temas em saúde (João Pessoa), $18(1), 143-170$.

Moraes, G. H. \& Albuquerque, E. M. (2019). As Estatísticas da Educação Profissional e Tecnológica: silêncios entre os números da formação de trabalhadores. Textos para discussão, $45,07-45$.

Moraes, M. C. L. (2017). Promoção da saúde: visitando conceitos e ideias. Revista Família, Ciclos de Vida e Saúde no Contexto Social, 5(1), 75-79. https://doi.org/10.18554/refacs.v5i1.1917.

Penteado, R. Z. \& Pereira, I. M. T. B. (2007). Qualidade de vida e saúde vocal de professores. Revista de Saúde Pública, 41, 236-243. https://doi.org/10.1590/S0034-89102007000200010.

Pereira, E. S., Abdala, G. A., Meira, M. D. D. \& Souza, A. C. (2017). Estilo de Vida e qualidade de vida de hipertensos em uma unidade básica de saúde. Revista Sodebras, 12(137), 99-103.

Pereira, A. S., Shitsuka, D. M., Parreira, F. J., Shitsuka, R. (2018). Metodologia da pesquisa científica. UFSM https://repositorio.ufsm.br/bitstream/handle/1/15824/Lic_Computacao_Metodologia-Pesquisa-Cientifica.pdf?sequence=1

Perrenoud, P. \& Thurler, M. G. (2007). As competências para ensinar no século XXI: a formação dos professores e o desafio da avaliação. Porto Alegre: Artmed Editora.

Rocha, S. V., Cardoso, J. P., Santos, C. A., Munaro, H. L. R., Vasconcelos, L. R. C. \& Petroski, E. L. (2015). Sobrepeso/obesidade em professores: prevalência e fatores associados. Revista Brasileira de Cineantropometria Desempenho Humano, 17(4),450-459. http://dx.doi.org/10.5007/1980-0037.2015v17n4p450.

Rodriguez-Añez, C. R., Reis, R. S. \& Petroski, E. L. (2008). Versão brasileira do questionário estilo de vida fantástico: tradução e validação para adultos jovens. Arquivos Brasileiros de Cardiologia, 91(2), 102-109. http://dx.doi.org/10.1590/S0066-782X2008001400006.

Santos, C. P., Dechandt, S. G., Bittencoust, J. A., Oliveira, J. M., Carvalho, C. \& Leite, C. E. (2016). Qualidade de vida no trabalho: um estudo sobre os professores da rede pública de ensino do Distrito Federal. Universitas. Gestão e Tecnologia, 6(1), 97-107. https://doi.org/10.5102/UN.GTI.V6I1.3875 
Research, Society and Development, v. 10, n. 3, e13110312878, 2021

(CC BY 4.0) | ISSN 2525-3409 | DOI: http://dx.doi.org/10.33448/rsd-v10i3.12878

Scagliusi, F. B., Polacow, V. O., Cordás, T. A., Coelho, D., Alvarenga, M., Philippi, S. T. \& Lancha Júnior, A. H. (2006). Tradução, adaptação e avaliação psicométrica da Escala de Conhecimento Nutricional do National Health Interview Survey Cancer Epidemiology. Revista de Nutrição, 19(4), 425-436. https://doi.org/10.1590/S1415-52732006000400002.

Silva, D. F. (2019). Jornada de trabalho, qualidade de vida e prática de atividades físicas de professores. Revista Sítio Novo, 3(1), 83-90. http://dx.doi.org/10.47236/2594-7036.2019.v3.i1.83-90p.

Silva, O. O. N., Ramos, M. D. P., Miranda, T. G., Bordas, M. A. G. (2019). Condições de trabalho docente: uma análise de Revistas de Educação da Bahia. Revista Tempos e Espaços em Educação, 12(28), 233-248. https://doi.org/10.20952/revtee.v12i28.8645.

Soares, L. J. S. \& Henig, E. V. (2019). Qualidade de vida no trabalho dos professores do ensino médio da rede pública e privada: o caso comparativo em cinco escolas de Rondonópolis Mato Grosso. Revista Estudos e Pesquisas em Administração, 3(2), 96-114. https://doi.org/10.30781/repad.v3i2.8554.

Tabeleão, V. P., Tomasi, E. \& Neves, S. F. (2011). Qualidade de vida e esgotamento profissional entre docentes da rede pública de Ensino Médio e Fundamental no Sul do Brasil. Cadernos de Saúde Pública, 27(12), 2401-2408. https://doi.org/10.1590/S0102-311X2011001200011.

Vedovato, T. G., Monteiro, M. I. Perfil sociodemográfico e condições de saúde e trabalho dos professores de nove escolas estaduais paulistas. Revista da Escola de Enfermagem da USP, 42(2), 291-297. https://doi.org/10.1590/S0080-62342008000200012.

WMA. World Medical Association. (2013) World Medical Association Declaration of Helsinki: ethical principles for medical research involving human subjects. JAMA, 310(20) 2191-2194.

Zaragoza, J. M. E. (1999). O mal-estar docente: a sala-de-aula e a saúde dos professores. Florianópolis: Edusc. https://doi.org/10.3333/ps.v3i3.234. 